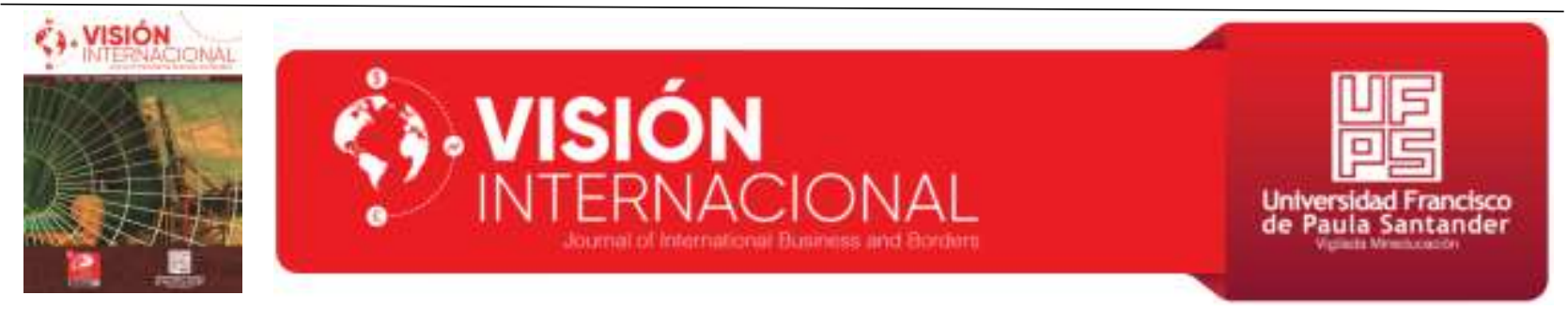

Artículo Original

DOI:https://doi.org/10.22463/27111121.2600

\title{
Oportunidades de negocios del mercado de frutas exóticas de Norte de Santander para los Emiratos Árabes Unidos
}

\author{
Business opportunities of the exotic fruit market of Norte de Santander for the United Arab \\ Emirates.
}
Ana Isabel Tamayo Medina ${ }^{1 *}$, Dayana Villamizar $^{2}$, Diego Chacon $^{3}$
1 *Magister en Administración de Empresas e Innovación, anaisabeltm@ufps.edu.co, ORCID 0000-0003-2583-811X, Universidad Francisco de Paula Santander, Cúcuta, Colombia.
2 Profesional en formación en Comercio Internacional, dayanacatherinevc@ufps.edu.co,Universidad Francisco de Paula Santander, Cúcuta, Colombia
3 Profesional en formación en Comercio Internacional, juandiegocr@ufps.edu.co, Universidad Francisco de Paula Santander, Cúcuta, Colombia

Cómo citar: Tamayo Medina, A. I., Villamizar, D., Chacon, D., \& Arenas, N. (2020). Oportunidades de negocios del mercado de frutas exóticas de Norte de Santander para los Emiratos Árabes Unidos. Visión Internacional (Cúcuta), 2(1), .

https://doi.org/10.22463/27111121.2600

Recibido: Febrero 15 ,2019 ; Aceptado: Marzo 03,2019

\begin{tabular}{|c|c|}
\hline & RESUMEN \\
\hline Palabras clave: & El departamento de Norte de Santander no es muy competitivo a nivel internacional debido a que su economía no ha sido \\
\hline Frutas Exóticas & bien administrada, es probable que sus gobernantes no efectúen aportes significativos a los diferentes sectores de la \\
\hline Emiratos Árabes Unidos & economía; uno de los sectores poco favorecidos es el agrícola, por ello el objetivo de la investigación es analizar las \\
\hline Economía & oportunidades de negocio que tiene el mercado Norte Santandereano. El tipo de metodología fue cuantitativa porque tiene \\
\hline Apertura Económica & criterios estadísticos basados en la capacidad productora que tiene Norte de Santander con el fin de lograr convertirse en un \\
\hline Oportunidad de Negocio & competidor a nivel internacional en el sector de frutas exóticas y el método fue descriptivo, ya que se refiere a la capacidad \\
\hline \multirow[t]{3}{*}{ Inversión } & e ineficiencia a la hora de alcanzar grandes metas de producción de los agricultores de Norte de Santander. La conclusión \\
\hline & $\begin{array}{l}\text { es que, si Norte de Santander logra realizar acuerdos en el cual los gobernantes se comprometan a ayudar con inversión al } \\
\text { sector agrícola. }\end{array}$ \\
\hline & ABSTRACT \\
\hline Keywords: & The department of Norte de Santander is not very competitive at the international level because its economy has not been \\
\hline Exotic Fruits & well managed, it is probable that its leaders do not make significant contributions to the different sectors of the economy; \\
\hline United Arab Emirates & one of the underprivileged sectors is agriculture, therefore the objective of the research is to analyse the business \\
\hline Economy & opportunities that the North Santanderean market has. The type of methodology was quantitative because it has statistical \\
\hline Economic Openness & criteria based on the production capacity that Norte de Santander has in order to become an international competitor in the \\
\hline Business Opportunity & exotic fruit sector and the method was descriptive, since it refers to the capacity and inefficiency in reaching large \\
\hline Investment. & production goals for farmers in Norte de Santander. The conclusion is that, if Norte de Santander manages to reach \\
\hline & $\mathrm{h}$ the rulers commit to help the agricultural sector with investment. \\
\hline
\end{tabular}

\section{Introducción}

Norte de Santander se caracteriza por la diversidad de municipios los cuales presentan innumerables ventajas en la producción de frutas exóticas gracias a sus variadas condiciones naturales que le permiten aprovechar sus tierras. Sin embargo, en términos relacionados con el comercio internacional norte de Santander ha tenido una participación limitada, que ha sido

*Autor de Correspondencia.

E-mail: anaisabeltm@ufps.edu.c (Ana Isabel Tamayo)

(c) $\Theta \Theta$ Peer review is the responsibility of the Universidad Francisco de Paula Santander

This is an article under the license CC BY-ND 
aprovechada por otros departamentos, en los procesos de comercialización de nuestros productos, así pues, las condiciones de logística de esos otros departamentos a nivel de comercio internacional le son favorables en sus procesos de intercambio comercial, y negociación.

Según la Opinión (2019), en la Provincia de Ocaña se está trabajando con productores de frutas frescas (gulupa, uchuva, granadilla, maracuyá y lulo), que requieren un acompañamiento para exportar de manera directa. Dichos agricultores no han tenido una ayuda para buscar nuevos mercados internacionales, por ello la importancia de buscar oportunidades para negociación en los emiratos árabes unidos. El departamento cuenta con un gran apoyo de parte del sector primario en la economía de la región; es de suma importancia fortalecer dicho sector para la mejora de su participación en el PIB nacional.

En lo reflejado anteriormente podemos decir también, que es de preocupación desde hace algún tiempo la situación reflejada por nuestros exportadores colombianos al tratar de establecer una relación constante y duradera con Emiratos Árabes, aunque las oportunidades para conseguir negocios están en todos los sectores, es por ello, que en la publicación de la revista dinero.com de marzo de 2012, según la Dra. Cecilia Porras Erazo, Presidente de la cámara de Comercio Árabe Colombiana expresa las oportunidades que tenemos en dichos mercados, con nuestros productos orgánicos y la demanda va en aumento según la Dra. Cecilia Porras Erazo.

En la economía del departamento Norte de Santander existen dos tipos de productores: el más común es el productor que vende la fruta en los meses de gran demanda a intermediarios, mientras que en los meses de baja demanda tiene dificultades en su comercialización por otro lado, se encuentra el productor que cuenta con más experiencia y organización, por lo que mantiene contratos de compra con las exportadoras garantizando ventas permanentes a un precio establecido por esta razón actualmente se ve la importancia o necesidad de comercializar no solo un producto en bruto que tenga ciclos de bajas y altas ventas, sino de ofrecer un valor agregado que se comercialice constantemente. Según Cárdenas,
A. Y. L., Paredes, Y. C. V., \& Verjel, M. S. G. (2018).

La siguiente investigación tiene como fin determinar las oportunidades de negocio que tienen los agricultores de Norte de Santander en los emiratos árabes unidos para la internacionalización de las frutas como el lulo, gulupa y uchuva que son producidas por la región y que no son aprovechadas en su mayoría. Las frutas exóticas son apetecidas internacionalmente gracias al gran contenido de vitaminas, minerales y demás beneficios que ofrecen como lo son su atractivo sabor y su diversidad de colores.

\section{PROBLEMÁTICA}

Norte de Santander es catalogada por el (Departamento Administrativo Nacional De Estadística - DANE. 2017) como el tercer departamento con más pobreza monetaria y ocupa un cuarto lugar como la región con más desempleo en Colombia. Lo cual significa que en la región fronteriza no hay crecimiento económico y puede que sus ingresos no sean aptos para realizar inversiones en gran magnitud en los diferentes sectores económicos del departamento. Como consecuencia a la problemática, el departamento tiene una desventaja en la competitividad ante los demás departamentos para obtener oportunidades de negocios de talla internacional el cual representa una mejora en el PIB regional de norte de Santander.

Según el plan de desarrollo de Norte de Santander un norte productivo para todos (2016) indica que el Departamento se encuentra ubicado en la parte nororiental de Colombia; con una extensión de $21648 \mathrm{~km}^{2}$, representando el 1.89\% del territorio nacional. El cual por su extensión de tierra es rico en la producción agrícola según él (instituto geográfico Agustín Codazzi, 2016) afirma que el 6,5\% de Norte de Santander cuenta con suelos aptos para cultivar, pero tiene un $40 \%$ con suelos afectados. De este modo podemos determinar cómo fiel competidor en mercados internacionales al departamento con respecto a las otras regiones del país es que se centre en la mejora de su producción de productos agrícolas y aprovechen al máximo los beneficios para aumentar la economía en el sector agrícola. 
Según REYES y otros (2006). Plan frutícola nacional. "el área está representada por 26 especies; que presentan en la mayoría de los casos rendimientos promedio por debajo de los obtenidos a nivel nacional".

Esto representa que norte de Santander tiene como tal una gran demanda de frutas que lo pueden catalogar como proveedor a una comercializadora internacional y no la está aprovechando y le está dejando el camino libre a los demás departamentos para que desarrollen, ventajas al momento de realizar una exportación de gran magnitud de productos agrícolas en este caso las frutas exóticas.

Según Villamizar (2018) indica que "los productores envían hasta 12 toneladas de uchuva y 6 toneladas de gulupa". Esto significa que Norte de Santander tiene la capacidad de realizar un amplio proceso de exportación a otros mercados potenciales que le garanticen la mejor comercialización de sus productos agrícolas ya que por medio de dicha dinámica la economía en el sector agrícola en la región se mejoraría en términos generales en competitividad con respecto a otras regiones del país que ya se encuentran en proceso de exportación a gran escala.

Debido a que norte de Santander representa gran cantidad de producción de frutas exóticas en los diferentes municipios de la región se puede expandir a los mercados potenciales que tengan un alto consumo de frutas exóticas en este caso las uchuvas y la gulupa según Colprensa (2018) indica que por medio de la visita del viceministro a los emiratos árabes unidos se abre una gran Oportunidad de negocio a los productores del país por que el interés de los EAU se centra por la gran demanda de consumo de productos agrícolas especialmente en las frutas frescas y exóticas, con respecto a lo anterior se visualiza que la ventaja que tiene norte de Santander es tener la capacidad de realizar inversiones que le favorezcan al incremento de la producción certificada y tecnificada de frutas frescas y en este caso frutas exóticas tales como la uchuva y gulupa.

\section{Resultados de la investigación}

\section{Situación de las frutas exóticas en Colombia y Norte de Santander}

Según (Colombia productiva, 2017) Colombia tiene ventajas competitivas en el sector agrícola, debido a la ubicación geográfica y a sus diferentes condiciones de suelo y clima, también a la identificación de zonas optimas que cumplen con los requisitos para la producción, lo que le permite tener una producción constante durante todo el año y facilitar el abastecimiento al mercado interno y proyectarse a la apertura económica; gracias al clima y el suelo los agricultores tienen una fuerte presencia, pero hay departamentos que casi no gozan de estos beneficios como es el caso de Norte de Santander que se limita en sus producciones, ya que los agricultores no tienen los conocimientos ni técnicas innovadoras en los proceso para la manipulación y el cultivo, porque son tradicionales lo cual desfavorece la producción.

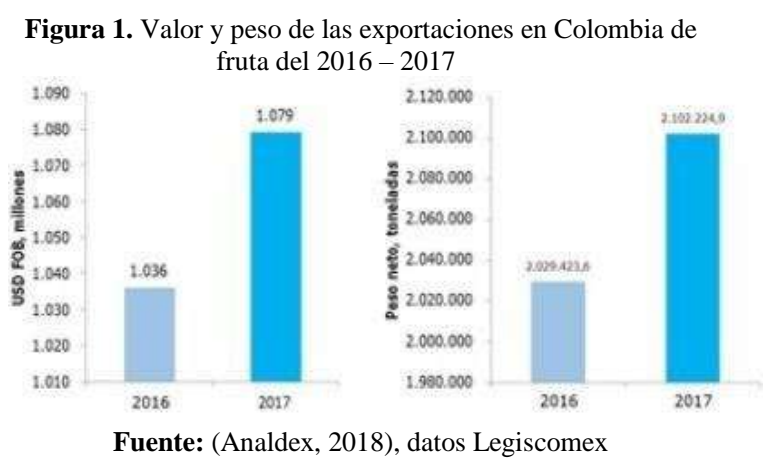

Colombia detona un incremento en las exportaciones de frutas, creciendo del 2016 al 2017 en un $4.15 \%$, con respecto al valor FOB este en el 2016 fue de 1.036 .040 millones exportador y en el 2017 de 1.079.110 millones, y en términos de porcentaje fue de $3.3 \%$ a $2.8 \%$ respectivamente, incrementando sus exportaciones en un 5\%, cabe resaltar que en el año 2017se exportaron 2.100.000 de toneladas de fruta. (Analdex, 2018) 
Figura 2. Valor de las exportaciones en el 2017 de las 5 frutas más importantes

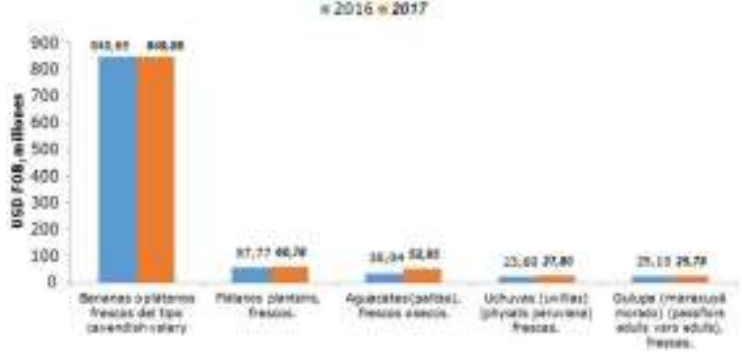

Fuente: (ANALDEX, 2018), datos Legiscomex

Entre las cinco frutas más importantes de las exportaciones de Colombia se encuentra las frutas exóticas que son las uchuvas y la gulupa, aunque son las dos de menor valor, puede resultar algo beneficioso para Norte de Santander, ya que la investigación busca analizar las oportunidades que se tiene al exportar fruta exótica a Emiratos Árabes Unidos, el cual es un destino con el que no se ha negociado, según la tabla 1 , y podría ser asertivo en las exportaciones de frutas de Colombia, ya que demanda este producto porque no lo produce; así mismo se puede notar en el grafico 3, que norte de Santander solo exporta una pequeña parte de las frutas, ya que entra en la porción de "otros" el cual solo tiene $4 \%$ y se deriva de varios departamentos más.

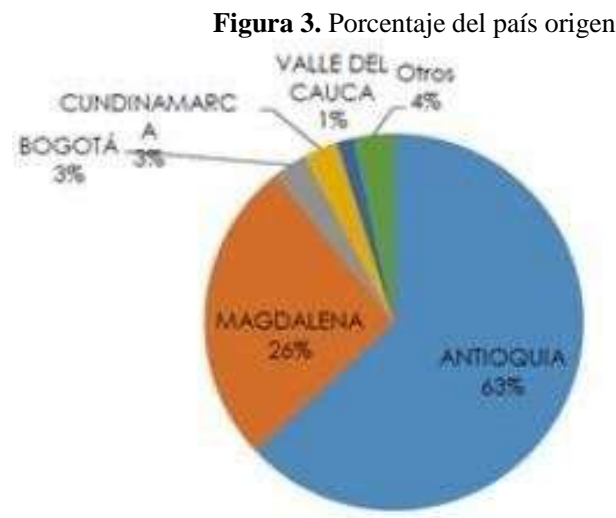

Fuente: (Analdex, 2018), datos Legiscomex
Tabla 1. Países destino de exportación de frutas en Colombia.

\begin{tabular}{|c|c|c|c|c|c|c|c|}
\hline \multirow[b]{2}{*}{$\begin{array}{l}\text { Pasise } \\
\text { pipen }\end{array}$} & \multicolumn{3}{|c|}{ Pesp (Tomilatis netes) } & \multicolumn{4}{|c|}{ Yilc (USp For Killowes) } \\
\hline & 2016 & 2017 & 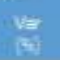 & 216 & 2017 & ats & Pat 2018 \\
\hline Bélgica & $518: 258,53$ & 59t. 695,49 & 2492 & 245 & $28 \mathrm{t}$ & $15:$ & 25,05 \\
\hline $\begin{array}{l}\text { Esbodos } \\
\text { Unides }\end{array}$ & 362817,57 & $348,377,54$ & Fth & 182 & 158 & $-10 \%$ & 15.646 \\
\hline Reino Unido & $396,071,02$ & $284.928,73$ & -254 & 196 & 153 & $-22 \%$ & $: 4.241$ \\
\hline Paises Bajos & $138.605,82$ & 158.004 .50 & 249 & 112 & 125 & $13:=$ & 11.736 \\
\hline Italia & $328.949,85$ & $2 \pi .506 .77$ & -1696 & 145 & 122 & -16 th & 11.396 \\
\hline Alemania & 139.425 .60 & \pm 4.193 .27 & $2 \mathrm{zi}$ & 58 & 6 & $-2 \%$ & 6.136 \\
\hline Esloveria & 12369,19 & 86966.91 & 6039 & 5 & 35 & 36984 & 3276 \\
\hline Esparia & $17.616,95$ & 32.454 .56 & 84th & 15 & 25 & 2095 & 235 \\
\hline Francia & $4.731,29$ & $18,090,35$ & $2828:$ & 7 & 14 & 9098 & $13 \%$ \\
\hline Grecia & 23.755 .79 & 31.00227 & $31 \%$ & 11 & 13 & $25 \%$ & 1256 \\
\hline Portogl & $9.459,92$ & $19.757,50$ & $10 \% 6$ & 5 & 10 & 1189: & $20 \%$ \\
\hline Suitra & $6.767,34$ & 18.482 .15 & 1736 & 4 & 10 & $139 \%$ & Q.96: \\
\hline Ecuador & 9.05470 & 20.585 .00 & $127 \%$ & 1 & 8 & $5 \times 3 \%$ & 0.785 \\
\hline Turquia & 2.673 .94 & 13.322 .22 & $390 \%$ i & 1 & 5 & $59 \%$ & $05 \%$ \\
\hline Suecia & 4.73489 & 10.531 .12 & $125 \mathrm{~s}$ & 2 & 5 & 245 & $0.5 \%$ \\
\hline Otros & 44.210 .82 & 49.255 .42 & $11 \%=$ & 33 & 38 & 2546 & 3.56 \\
\hline Tobal & $2.029423,59$ & $2.102 .224,93$ & 46 & 2.036 & 1.079 & An & 1004 \\
\hline
\end{tabular}

Fuente: (Analdex, 2018), datos Legiscomex

Pueden ser varias las variables por las que Norte de Santander no exporte una gran cantidad de frutas exóticas, como que los cultivos de varios sectores se utilicen para cultivos ilícitos, que no se especialice en la exportación sino en la producción o que no tengan la capacidad productiva, pero tampoco aprovecha el hecho de la comercialización porque hay departamentos que compran a otros departamentos los productos que necesitan para poder cumplir con la cantidad exigida por el cliente y exportarlo.

Por ende, a Norte de Santander en cualquiera de los tres casos dichos anteriormente les ayudaría la inversión por parte del gobierno a los agricultores hortofrutícolas, para que ayuden en materia de capacitaciones y estos abran su mentalidad no solo a la producción, sino a una comercialización mayor y al mercado internacional, también contribuirían a la tecnificación de los procesos de producción para que los agricultores puedan cumplir la demanda del mercado tanto nacional como internacional.

De acuerdo con el sector agropecuario (2011) “existían en el país 743 hectáreas destinadas al cultivo de uchuva con una producción total de 10.771 toneladas". En el año 2016 las hectáreas aumentaron significativamente pasando a 1.023.100 áreas cultivadas y 15.111 .780 toneladas como se muestra en la siguiente tabla, distribuida por departamentos. 
Tabla 2. Producción de uchuva en los departamentos de Colombia

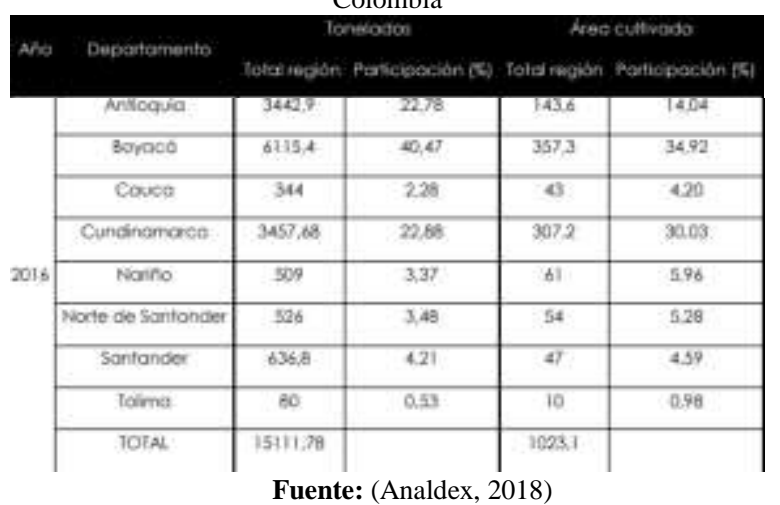

Al comparar las producciones de toneladas con las áreas cultivas de uchuva de cada departamento, se puede notar como Norte de Santander posee una gran cantidad de hectáreas respecto a otros departamentos pero lo que recolecta es poco y está entre los departamentos que menos áreas de cultivo tienen, por ende, menos producción, por ello es importante que los gobiernos contribuyan a los agricultores para que no desistan de este producto que podría generar el incremento en la economía del país, ya que, según la Opinión (2015) afirma que:

La exportación de uchuva y gulupa producida en los municipios de Silos, Cacota y Chitagá no se continuará efectuando porque los volúmenes de producción no son suficientes para cubrir la demanda del mercado externo. Los productores buscan agruparse en grandes asociaciones para obtener grandes volúmenes de frutas y conquistar el mercado internacional.

A su vez, el director de Asohofrucol Villamizar (2015) informo en un seminario que efectuó a la asociación:

"Estamos organizando a los productores y buscando apoyo de otras empresas exportadoras con el fin de retomar el mercado internacional, para ello creamos Frutinorte Export, que la conforman once asociaciones de los municipios productores de uchuva y gulupa, para retomar la comercialización e incorporar las frutas cítricas y el aguacate hazz."

Por otro lado, el director gerente general de Asohofrucol Palacio (2015) indico que "en el territorio nacional hay 921.000 hectáreas hortofrutícolas sembradas, equiparados en área con los cafeteros, y la producción nacional llega a
10,8 millones toneladas, es el segundo sector que emplea más mano de obra después del café".

\section{Agenda interina de productividad y competitividad enfocada a las frutas y hortalizas}

Debido a los vaivenes económico entre la frontera colombo - venezolana Norte de Santander necesitaba construir una base productiva que redujera su vulnerabilidad por la crisis fronteriza, por ello, se creó la agenda interina que está enfocada en las apuestas que hacen para el año 2020 en los sectores comerciales con el fin de que el país aproveche sus productos para generar un incremento en el intercambio comercial y así afrontar los riesgos que se han generado de la problemática fronteriza. La agenda interina está basada en dos factores, primeramente, la productividad ya que para aumentar el ingreso nacional per cápita, un país debe utilizar una mayor productividad en el uso de los recursos, lo que genera el segundo factor que es la competitividad, el cual ayuda a la población a mejorar sus ingresos y con esto su calidad de vida.

Una de las apuestas que se encuentra en el sector agroindustrial son las frutas y hortalizas, el objetivo de este sector es incrementar en Norte de Santander el área sembrada de frutales y mejorar la productividad por medio de las tecnologías de invernadero, teniendo como meta obtener 150 toneladas por hectárea, lo cual se puede cumplir, ya que el departamento cuenta con producción por todo el año y tiene ciertas ventajas competitivas como lo es el gremio Asohofrucol y las 14 organizaciones compuestas por numerosas familias campesinas, también los proyectos a largo plazo para incrementar la producción de frutales, aunque no solo se debe incrementar la producción sino también innovar dándoles valor agregado a los productos.

A su vez, se debe mejorar las técnicas de producción con respecto a las normas sanitarias, procurando obtener una producción más limpia y buenas prácticas agrícolas, ya que es indispensable para que estos productos accedan al mercado internacional, por otro lado, es importante aumentar la producción para que no se limite el desarrollo de una oferta permanente con algún país, por esto se debe cumplir una serie de necesidades que se plasman en el plan de 
competitividad y producción para las frutas y hortalizas.

Tabla.3 Plan de competitividad y productividad para las frutas y hortalizas.

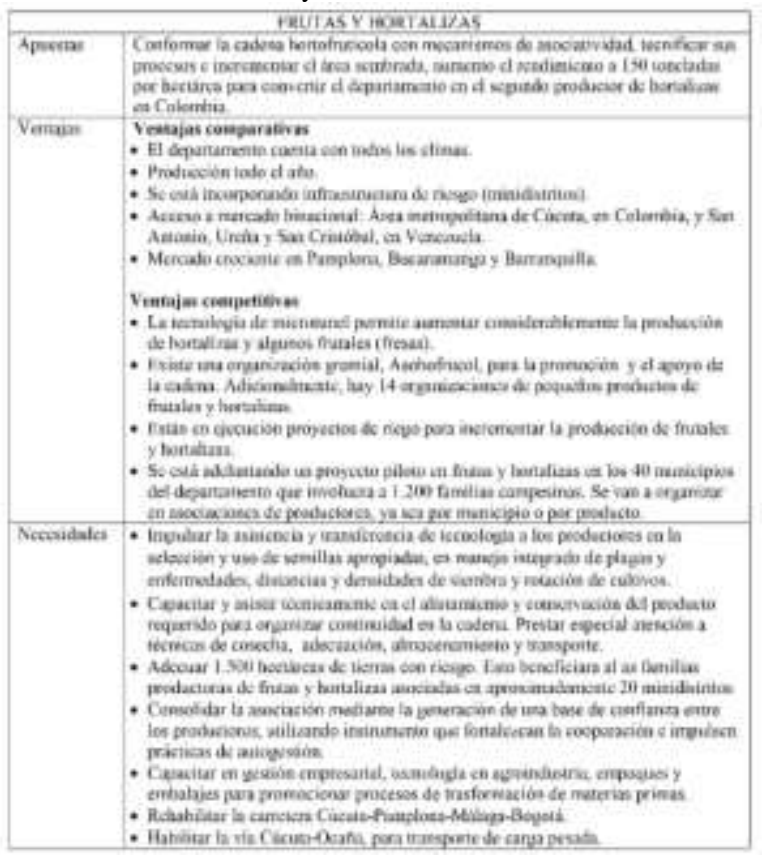

Fuente: Data Cúcuta.

Intereses de los Emiratos Árabes Unidos en productos agrícolas especialmente en productos exóticos o tropicales

El interés que se presenta en los habitantes y comerciantes de los estados pertenecientes de los EAU es muy importantes resaltarlo ya que se puede lograr generar excelentes oportunidades de negocio a nivel internacional establecer estrategias el cual ayude a los agricultores a tecnificar sus procesos y llevar acabo la comercialización de sus productos a nivel internacional según la república.2018 le realizo una entrevista al El viceministro de Asuntos Agropecuarios, Samuel Zambran el cual expreso que.

"Ellos están muy interesados en las frutas tropicales. Les manifestamos, que tenemos unas exportaciones nacientes pero muy importantes en el marco de los tratados de libre comercio, en frutas como: Lima Tahití, Aguacate Hass, Mango, Piña Golden, las Pasifloras (Maracuyá, Uchuva, Pitahaya), y otros productos que en estos países son muy atractivos"
Según con lo anterior se puede resaltar que tanto Colombia y los diferentes departamentos que lo conforman están siendo reconocido a nivel mundial por sus excelentes productos agrícolas el cual está dispuesto a ofrecer, debido a que los emiratos árabes unidos tiene ese grado de interés enfocado a los productos agrícolas es necesario iniciar con propuestas que les ayude a los agricultores tanto del país como los del departamento norte de Santander tengan el apoyo del gobierno con el fin de incentivar a que los procesos tengan más dedicación para generar productos de gran calidad .

\section{Conclusiones}

Norte de Santander cuenta con numerosas áreas que permiten en el sector agrícola el cultivo de productos, gracias a la ubicación geográfica y las diferentes condiciones del suelo y clima, lo que permite una constante producción anual, por ende, se necesita que los agricultores de Norte de Santander vean el comercio de una manera grande, no solo a nivel departamental sino que abran sus mentes a los mercados internacionales, capacitándose en darle valor agregado a sus productos, en este caso, a las frutas y hortalizas.

Por otro lado, también deben aprovechar que las exportaciones de las frutas exóticas que tanto le apetecen a los Emiratos Árabes Unidos no están siendo abastecidas, por ende, deben tecnificar sus procesos de producción o crear planes de comercialización para estar preparados y poder proveer una cantidad suficiente que llegue a ser demandada por los Emiratos Árabes, mientras los agricultores deben estar pendientes de los avances del plan de productividad y competitividad para aprovechar los beneficios que se vayan desarrollando, y así contribuir tanto en tecnología como recursos sus áreas de cultivo.

En definitiva, los emiratos árabes es un mercado con gran potencial para realizar oportunidades de negocios de talla internacional, en lo que se refiere a recursos de capital e inversión, más si se refiere al sector productivo, debemos como región tener en cuenta el impacto positivo para nosotros, si logramos una negociación estable, con unos procesos de exportación y logística acordes a nuestros 
productos y favoreciendo la economía del departamento de norte de Santander.

\section{Bibliografía}

Analdex. (2018). Comportamiento de la uchuva producción y comercio. En línea. https://www.analdex.org/wpcontent/uploads/2018/02/2018-02-08Mercado-de-la-Uchuva.pdf

Analdex. (2018). Informe de exportaciones e importaciones de fruta 2017. En línea.https://www.analdex.org/2018/04/1 6/informe-de-exportaciones-eimportaciones-de-fruta-2017/

COLPRENSA (2018). Emiratos Árabes quiere importar frutas colombianas. Bogotá. En líneahttps://www.elcolombiano.com/neg ocios/agro/emiratos-arabes-quiereimportar-frutas-decolombia-dc8127371

Departamento Nacional de Planeación. (2007). Agenda interna para la productividad y la competitividad. En línea.

http://www.datacucuta.com/PDF/publicacionesexternas/COMISIONREGIONALDECO MPETITIVIDAD/AGENDA\%20INTER NA\%20DE\%20COMPETITIVIDAD.pd f

La Opinión. (2015). Baja producción de uchuva y gulupa frena su exportación. En línea.https://www.laopinion.com.co/econ omia/baja-produccion-de-uchuva-ygulupa-frena-su-exportacion-102650\#OP

Legiscomex. (2013). Exportación de Frutas Exóticas. En línea. https://www.legiscomex.com/BancoMed ios/Documentos\%20PDF/estudio-frutasexoticas-colombia-completo.pdf

REYES Y OTROS. (2006). Plan frutícola nacional. Cúcuta. En línea. https://www.google.com/search?q=asoh ofrucol+norte+de+santander\&oq=asofrui cola\&aqs=chrome.2.69i57j015.13374j1j7 \&sourceid $=$ chrome \&ie $=$ utf -8

VILLAMIZAR. (2018). La opinión. Cúcuta. En línea

https://www.laopinion.com.co/economia /uchuva-y-gulupa-de-cacota-conquistaneeuu-yalemania-161196\#op

VILLAMIZAR. (2019). La opinión. Cúcuta. En línea

https://www.laopinion.com.co/economia /arroz-aceite-de-palma-frutas-y-cafelideraranexportaciones-de-la-region169533

(2016.) Plan de desarrollo para Norte de Santander "un norte productivo para todos. Cúcuta. En línea http://www.sednortedesantander.gov.co/ sitio/images/documentos/informesdelsec tor/pdd\%20nds \%202016-2019.pdf

(2016). 6,5\% de Norte de Santander cuenta con suelos aptos para cultivar. Bogotá. En línea.https://noticias.igac.gov.co/es/conte nido/65-de-norte-de-santander-cuentacon-suelos-aptos-paracultivar

La república. 2018 entrevista al viceministro de Asuntos Agropecuarios, Samuel Zambrano sobre el interés de los emiratos árabes unidos en los productos agro colombiano. En línea https://www.larepublica.co/economia/em iratos-arabes-unidos-esta-interesado-enimportar-productos-del-agrocolombiano-2595548

Cárdenas, A. Y. L., Paredes, Y. C. V., \& Verjel, M. S. G. (2018). Propuesta para aumentar la competitividad de los productores de Uchuva de Norte de Santander mediante la diversificación de su oferta exportable. Aibi revista de investigación, administración e ingeniería, 3949.https://revistas.udes.edu.co/aibi/articl e/view/484 